Bull. Fac. Agric., Cairo Univ., 59 (2008):13-23.

\title{
EVALUATION OF SOME EGYPTIAN COTTON GENOTYPES IN THE FIRST AND THE SECOND PICKS BY LATIN SQUARE DESIGN WITH A COMBINED ANALYSIS
}

(Received: 8.4.2007)

\author{
By \\ H. A. Idris \\ Cotton Research Institute, Agricultural Research Center, Giza, Egypt.
}

\begin{abstract}
The present investigation is dealing with the variances of five long staple Egyptian cotton (Gossypium barbadense L.) genotypes, with respect to yield, its components and fiber properties in the first and the second picks in Delta (Gharbia and Mounofia). Genotypes were three cultivars, viz. G.85, G.86 and G.89, which are normally grown in Delta. The others were hybrids, viz (G.89 x G.86) and (G.89 x Pima S-6). One field experiment latin square design was carried out in the two locations. Results of simple latin square exhibited that G.89 x Pima S-6 was the best genotype with respect to yields (seed and lint) since it kept the first rank in the first pick in the two locations and the maturity of this hybrid was faster than other genotypes. G.86 was more skilled with respect to fiber length in the two picks in the two locations. Two analyses of combined latin square to estimate the environments variance in the first and the second pick by two ways direct and indirect. First analysis (direct) depends on two combined, one the first pick (two environments) and the other second pick (two environments). Second analysis (indirect) depends on one combined (four picks) and partitioning of pick. Direct depends on two analyses of combined to estimate environments variance in the first pick and the second pick. Indirect surpassed direct due to it needed one analysis of combined to estimate environments variance in the first pick and the second pick. Although direct and indirect calculated the same values of environments variance in the first pick and the second pick but they exhibited different results of significant variation due to different values of $F$ tabulated of them, which depends on degrees of freedom of error and different experimental error.
\end{abstract}

Key words: cotton, delta, environments, first pick, genotype, latin square, locations, second pick.

\section{INTRODUCTION}

Latin square design the randomization of treatments is restricted further by grouping them into columns as well as rows. Thus it is possible to remove variability from experimental error associated with both these effects. Each treatment occurs the same number of times (usually once) in each row and column. The design will afford a more precise comparison of treatment effects than the randomized block design only if there is appreciable variation associated with the columns, (Little and Hills, 1978). In this design layout of experiment is divided into homogenous blocks in two ways. The blocks in one direction are commonly known as rows and the blocks in other direction as columns. The number of plots in each row is the same as the number of plots in each column. This number is equal to the number of treatments, (Sing and Narayanan, 2000).

El Shaer et al., (1984) noticed that lint percentage increased at the last pick than at the other three ones. Abou Tour et al., (1996) evaluated five long staple cultivars, viz. G.75, G.81, G.85, G.86 and G.89 in Delta (El Sharkia, EL Gharbia and El Dakahlia). They used latin square design in each location. Results of the first pick revealed significant differences due to cultivars were observed with respect to boll weight, seed index, lint percentage, fiber length and micronaire value in the individual locations except boll weight in El Gharbia and micronaire reading in other two locations. Combined analysis exhibited significant differences for seed index, lint percentage and fiber length. Badr and El Sayed (2004) evaluated five long staple genotypes, viz. G.85, G.86, G.89, G.89 x G.86 and G.89 x Pima S-6 in Delta (El Sharkia, EL Gharbia and El Mounofia). Genotypes exhibited significant differences with respect to three yield components, viz. boll weight, seed index and lint percentage in the first pick. On the other hand, genotypes revealed non-significant differences for total seed cotton yield (sum of the two picks). Idris and Abd El-Rahman (2006) evaluated five 
long staple genotypes in two picks and two locations. They used analysis more than one observation per experimental unit for latin square design in each location. Results indicated that the variance between the first and second picks was more strongly influenced by environments due to different responses of genotypes in each location during the two seasons.

Researchers need a statistical measure to evaluate genotypes under the first and second picks. The objective of the present study was to evaluate genotypes in the first and second picks for some Egyptian cotton genotypes using latin square design and multiple regression.

\section{MATERIALS AND METHODS}

One field experiment $(5 \times 5)$ latin square design was carried out in two locations in Delta (Gharbia and Mounofia). Five long staple of Egyptian cotton (Gossypium barbadense L.) genotypes were grown. Three of them were cultivars, viz. G.85, G.86 and G.89, which are normally grown in Delta. The two remainders were hybrids, viz. (G.89 x G.86) and (G.89 x Pima S-6).). Planting was during the last week of March. All other agricultural practices were done as usual. Two picks (first and second) were taken in each experiment.

Genotypes were evaluated respecting yield and its components during 2005 and 2006 seasons in Gharbia and Mounofia, respectively. They were evaluated respecting fiber properties during 2004 and 2005 seasons in Mounofia and Gharbia, respectively. Explain that the program of Cotton Research Institute is continuous in each location a long time. Yield was seed cotton yield (S.C.Y.) kentar/ feddan (k/fed)) and lint cotton yield (L.C.Y.) kentar/ feddan (k/fed)). Yield components were (boll weight (B.W.) gm, lint percentage (L.P. \%), seed index (S.I.) gm and lint index (L.I.) gm. Fiber properties were fiber length (F.L) $\mathrm{mm}$ and micronaire reading (Mic.).

\subsection{Statistical analysis}

\subsubsection{Simple and combined latin square design}

The simple latin square was carried out with data of the two picks as previously mentioned in the two successive locations. Two analysis of combined latin square to estimate the environments variance in the first and the second pick by two ways direct and indirect. First analysis (direct) depends on two combined one the first pick (two environments) and one the second pick (two environments). Second analysis (indirect) depends on one combined (four picks) and partitioning of picks. Homogeneity test of variances (Bartlett test) was used according to procedures reported by Bailey (1994).

Statistical analysis was straightforward as Cochran and Cox (1950), Federer (1955), Gomez and Gomez (1984) and Roger (1994). The treatment means were compared by L.S.D. test as given by Steel and Torrie (1980). All comparisons were done at 0.05 level of significance. *, ** Significant at 0.05 and 0.01 levels, respectively. ---, Not significant at $5 \%$.

\subsubsection{Multiple regression}

To illustrate partial and multiple correlation and regression. Yields (seed and lint) $(\mathrm{Y})$, yield components $\left(\mathrm{X}_{1}\right)$ and $\left(\mathrm{X}_{2}\right)$ in the first pick and the second pick for simple and multiple latin square were analyzed (Table 1). Statistical analysis was straightforward as Little and Hills (1978).

*, ** Significant at 0.05 and 0.01 levels, respectively.

\section{RESULTS AND DISCUSSION}

3.1. Simple latin square design

The analysis of variance in the two picks in

Table (1): Multiple regression analysis of yields (seed and lint) (Y), yield components $\left(\mathrm{X}_{1}\right)$ and $\left(\mathrm{X}_{2}\right)$ for simple and combined latin square (two picks).

\begin{tabular}{|c|c|c|c|}
\hline \multirow[b]{2}{*}{ Source of variation } & \multicolumn{2}{|c|}{ d.f. } & \multirow{2}{*}{$\begin{array}{c}\text { Method of computing } \\
\text { SS }\end{array}$} \\
\hline & Simple & combined & \\
\hline $\begin{array}{l}X_{1} \text { Considered first } \\
\text { Total } \\
\text { Regression due to }\left(X_{1}\right) \\
\text { Deviation from simple regression } \\
\text { Additional regression due to }\left(X_{2}\right) \\
\text { Deviation from multiple regression }\end{array}$ & $\begin{array}{l}4 \\
1 \\
3 \\
1 \\
2\end{array}$ & $\begin{array}{l}9 \\
1 \\
8 \\
1\end{array}$ & $\begin{array}{l}\sum y^{2} \\
r_{y \times 1}^{2}\left(\sum y^{2}\right) \\
\left(1-\mathbf{r}_{y \times 1}^{2}\right) \sum y^{2} \\
r_{y \times 2 . x 1}^{2}\left(1-r_{y \times 1}^{2}\right) \sum y^{2} \\
\left(1-R_{v . \times 1 \times 2}^{2}\right) \sum y^{2}\end{array}$ \\
\hline $\begin{array}{l}X_{2} \text { Considered first } \\
\text { Total } \\
\text { Regression due to }\left(X_{2}\right) \\
\text { Deviation from simple regression } \\
\text { Additional regression due to }\left(X_{1}\right) \\
\text { Deviation from multiple regression }\end{array}$ & $\begin{array}{l}4 \\
1 \\
3 \\
1 \\
2\end{array}$ & $\begin{array}{l}9 \\
1 \\
8 \\
1\end{array}$ & 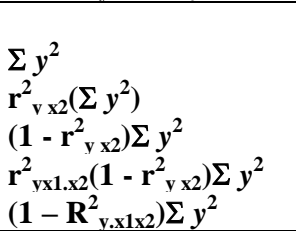 \\
\hline
\end{tabular}


each location revealed the presence of significance rows, columns, genotypes and partitioning of genotypes, (Table 2).

\subsubsection{Gharbia}

In the first pick, significant variation due to genotypes was observed for yields (seed and lint), yield components and fiber properties except seed index. Significant variation due to cultivars was recorded for yield components and fiber properties except seed index. Significant variation due to hybrids was observed for lint cotton yield, boll weight, fiber length and micronaire reading. Significant variation due to cultivars vs. hybrids was detected for yields (seed and lint), (Table 2).

G.89 x Pima. S-6 significantly surpassed all genotypes; with respect to yields (seed and lint) except the other hybrid G.89 x G.86 for seed cotton yield. G.86 had the highest values for boll weight, lint index and fiber length, it did not significantly differ from other genotypes except G. 85 and G.89 x Pima. S-6 for three characters, G.89 for lint index, significantly exceeded other genotypes., G. 85 did not significantly differ from G.86 and G.89 x Pima. S-6, significantly surpassed other genotypes; with respect to lint percentage. G. 89 x G.86 had the highest values for micronaire reading, it significantly surpassed all genotypes except G.89 and G.86, (Table 3).

In the second pick, significant variation due to genotypes was recorded for all characters. Significant variation due to cultivars was observed for yield components and fiber properties. Significant variation due to hybrids was observed for boll weight, seed index, lint index and fiber length. Significant variation due to cultivars vs. hybrids was detected for yields (seed and lint), (Table 2).

G.89 significantly exceeded other genotypes with respect to yields (seed and lint) and micronaire reading except G.85 for lint cotton yield, G.89 x G.86 for micronaire reading and G.86 for the three traits. G.86 had the highest values with respect to yield components and fiber length, it significantly surpassed other genotypes except G.89 and G.89 x Pima S-6 for boll weight, seed index and fiber length. It exceeded other genotypes except G.85 and G.89 x G.86 for lint percentage and lint index, respectively (Table 3 ).

These results exhibited that G.89 x Pima S-6 was the best genotype with respect to yields (seed and lint) since it kept the first rank in the first pick and the last rank in the second pick. Explain that the maturity of this hybrid was faster than other genotypes. G.86 was more skilled with respect to fiber length in the two picks. These results indicate that genotypes differently reacted in each pick except G.86 for fiber length.

\subsubsection{Mounofia}

In the first pick, significant variation due to genotypes was observed for yields (seed and lint), yield components and fiber properties. Significant variation due to cultivars was recorded for all traits except seed index and micronaire reading. Significant variation due to hybrids was observed for boll weight, fiber length and micronaire reading. Significant variation due to cultivars vs. hybrids was detected for yields (seed and lint), its components and fiber properties except lint percentage and micronaire reading, (Table 2).

In the first pick, G.89 x Pima S-6 gave similar results in Gharbia with respect to yields (seed and lint) due to it had the highest values for yield, significantly exceeded other genotypes except G. 89 x G.86. On the other hand, G.89 x G.86 gave the highest values for boll weight, seed index, lint index and micronaire reading, it significantly surpassed all other genotypes except G.89 x Pima S-6 for seed index, G.89 for micronaire reading and G.86 for the last two traits. G.85 gave the highest values for lint percentage, it did not significantly differ from G.86 and G.89 x Pima S6 significantly surpassed all other genotypes. G.86 was the best with respect to fiber length, it significantly exceeded other genotypes, (Table 3).

In the second pick, significant variation due to genotypes was detected for yields (seed and lint), yield components and fiber properties except boll weight and micronaire reading. Significant variation due to cultivars was observed for lint percentage, seed index, lint index and fiber length. Significant variation due to hybrids was recorded lint percentage. Significant variation due to cultivars vs. hybrids was detected for yields (seed and lint) and its components except boll weight and lint percentage, (Table 2).

In the second pick, G.89 gave the same results in Gharbia with respect to yields (seed and lint), due to it had the highest values for yield, and significantly exceeded other genotypes except G.85 and G.86. On the other hand, G.85 did not significantly differ from G.86 and G.89 x Pima S6 significantly surpassed all other genotypes for lint percentage. G.89 x G. 86 significantly exceeded all genotypes except G.89 x Pima S-6 and G.86 for seed index. G.89 x Pima S-6 significantly surpassed all genotypes except G.89 x G.86 and G.86 for lint index. G.86 was the best genotype for fiber length, it significantly surpassed all genotypes, (Table 3). 
Table (2): Mean squares of yield, its components and fiber for simple latin square.

\begin{tabular}{|c|c|c|c|c|c|c|c|}
\hline \multicolumn{8}{|c|}{$\begin{array}{rr}\text { First pick } \\
\end{array}$} \\
\hline \multicolumn{2}{|l|}{ Traits } & \multicolumn{2}{|c|}{ S. C. Y. (k/fed) } & \multicolumn{2}{|c|}{ L. C. Y. (k/fed) } & \multicolumn{2}{|c|}{ B. W. (gm) } \\
\hline Source of variation & d.f. & Gharbia & Mounofia & Gharbia & Mounofia & Gharbia & Mounofia \\
\hline Rows & 4 & 1.44 & $3.87 * *$ & 2.21 & $6.23 * *$ & 0.008 & 0.031 \\
\hline Columns & 4 & $3.53 * *$ & 0.678 & $5.35 * *$ & 1.31 & 0.016 & $0.084 * *$ \\
\hline Genotypes & 4 & $5.48 * *$ & 23.58** & $8.20 * *$ & $33.72 * *$ & $0.233 * *$ & 0.170** \\
\hline Cultivars $(\mathrm{C})$ & 2 & 0.681 & $4.89 * *$ & 2.08 & $8.48 * *$ & $0.220 *$ & $0.063 *$ \\
\hline Hybrids (H) & 1 & 2.84 & 0.936 & $\mathbf{5 . 3 4} *$ & 2.13 & 0.324* & $0.372 * *$ \\
\hline C. vs $\mathrm{H}$. & 1 & $17.73 * *$ & $83.63 * *$ & $23.28 * *$ & $115.79 * *$ & 0.169 & $0.180 * *$ \\
\hline Experimental error & 12 & 0.612 & 0.402 & 0.873 & 0.619 & 0.036 & 0.015 \\
\hline Source of variation & d.f. & \multicolumn{2}{|c|}{ L.P. $(\%)$} & \multicolumn{2}{|c|}{ S. I. $($ gm) } & \multicolumn{2}{|c|}{ L. I. (gm) } \\
\hline Rows & 4 & 0.150 & 0.373 & 0.672 & 0.976 & $0.305 *$ & $0.602 *$ \\
\hline Columns & 4 & 0.129 & 0.371 & 0.013 & 0.387 & 0.013 & 0.199 \\
\hline Genotypes & 4 & $5.09 * *$ & $2.85 *$ & 0.745 & $3.66 * *$ & $0.475 * *$ & $1.35 * *$ \\
\hline Cultivars $(\mathrm{C})$ & 2 & $9.25 * *$ & $3.96 *$ & 0.883 & 1.28 & $0.894 * *$ & $1.20 * *$ \\
\hline Hybrids (H) & 1 & 1.02 & 0.712 & 0.930 & 0.259 & 0.108 & 0.007 \\
\hline C. vs $\mathrm{H}$. & 1 & 0.831 & 2.74 & 0.283 & $11.81 * *$ & 0.003 & $2.99 * *$ \\
\hline Experimental error & 12 & 0.500 & 0.632 & 0.389 & 0.352 & 0.074 & 0.159 \\
\hline Source of variation & d.f. & \multicolumn{2}{|c|}{ F.L. $(\mathbf{m m})$} & \multicolumn{2}{|c|}{ Mic. } & & \\
\hline Rows & 4 & 0.622 & 0.115 & 0.049 & 0.042 & & \\
\hline Columns & 4 & 0.433 & 0.077 & 0.021 & 0.046 & & \\
\hline Genotypes & 4 & $9.69 * *$ & $5.28 * *$ & $0.234 *$ & $0.314 *$ & & \\
\hline Cultivars $(\mathbf{C})$ & 2 & $11.31 * *$ & $6.51 * *$ & $0.296 *$ & 0.115 & & \\
\hline Hybrids (H) & 1 & $15.62 * *$ & $2.12 *$ & $0.324 *$ & $1.02 * *$ & & \\
\hline C. vs $\mathrm{H}$. & 1 & 0.505 & $6.00 * *$ & 0.022 & 0.004 & & \\
\hline Experimental error & 12 & 0.416 & 0.292 & $\mathbf{0 . 0 5 3}$ & 0.069 & & \\
\hline \multicolumn{8}{|c|}{$\begin{array}{l}\text { Second pick } \\
\end{array}$} \\
\hline \multicolumn{2}{|l|}{ Traits } & \multicolumn{2}{|c|}{ S. C. Y. (k/fed) } & \multicolumn{2}{|c|}{ L. C. Y. (k/fed) } & \multicolumn{2}{|c|}{ B. W. (gm) } \\
\hline Source of variation & d.f. & Gharbia & Mounofia & Gharbia & Mounofia & Gharbia & Mounofia \\
\hline Rows & 4 & $1.94 * *$ & $0.504 *$ & $3.08 * *$ & $0.933 *$ & $0.079 *$ & 0.010 \\
\hline Columns & 4 & $0.227 * *$ & $0.769 *$ & $0.335 * *$ & $1.63 * *$ & 0.014 & 0.043 \\
\hline Genotypes & 4 & $0.767 * *$ & $0.511 * *$ & $1.23 * *$ & 0.859* & $0.097 * *$ & 0.021 \\
\hline Cultivars (C) & 2 & 0.133 & 0.063 & 0.100 & 0.386 & 0.089* & 0.010 \\
\hline Hybrids (H) & 1 & 0.062 & 0.014 & 0.086 & 0.090 & $0.132 *$ & 0.058 \\
\hline C. vs $\mathrm{H}$. & 1 & $2.74 * *$ & $1.90 * *$ & $4.62 * *$ & $2.57 * *$ & 0.080 & 0.006 \\
\hline Experimental error & 12 & 0.035 & 0.143 & 0.054 & 0.192 & 0.017 & 0.019 \\
\hline Source of variation & d.f. & \multicolumn{2}{|c|}{ L. P. $(\%)$} & \multicolumn{2}{|c|}{ S. I. (gm) } & \multicolumn{2}{|c|}{ L. I. (gm) } \\
\hline Rows & 4 & 0.188 & 0.717 & 0.390 & 1.20 & 0.194 & $\mathrm{0.805}^{*}$ \\
\hline Columns & 4 & 0.249 & 0.575 & 0.214 & 0.425 & 0.114 & 0.194 \\
\hline Genotypes & 4 & $4.55 * *$ & $3.97 * *$ & $2.07 * *$ & $3.22 * *$ & $1.22 * *$ & $1.58 * *$ \\
\hline Cultivars $(\mathrm{C})$ & 2 & $8.73 * *$ & $4.88 * *$ & $2.52 * *$ & $1.62 *$ & $1.80 * *$ & $1.44 * *$ \\
\hline Hybrids (H) & 1 & 0.092 & $5.16 * *$ & $3.23 * *$ & 0.306 & $1.23 * *$ & 0.028 \\
\hline C. vs $\mathrm{H}$. & 1 & 0.675 & 0.940 & 0.006 & $9.34 * *$ & 0.070 & $3.43 * *$ \\
\hline Experimental error & 12 & 0.586 & $\mathbf{0 . 3 9 7}$ & 0.221 & 0.385 & 0.102 & 0.186 \\
\hline Source of variation & d.f. & \multicolumn{2}{|c|}{ F.L. $(\mathbf{m m})$} & & & & \\
\hline Rows & 4 & 0.974 & 1.55 & 0.051 & 0.073 & & \\
\hline Columns & 4 & 0.240 & 0.602 & 0.047 & 0.055 & & \\
\hline Genotypes & 4 & $10.90 * *$ & $7.54 * *$ & $0.302 * *$ & 0.140 & & \\
\hline Cultivars $(\mathbf{C})$ & 2 & $14.26 * *$ & $12.43 * *$ & $0.504 * *$ & 0.139 & & \\
\hline Hybrids (H) & 1 & $14.64 * *$ & $2.92 *$ & 0.169 & 0.081 & & \\
\hline C. vs $\mathrm{H}$. & 1 & 0.417 & 2.35 & 0.029 & 0.202 & & \\
\hline Experimental error & 12 & 0.935 & 0.567 & 0.047 & 0.062 & & \\
\hline
\end{tabular}

These results exhibit that G.89 x Pima S-6 was the best genotype with respect to yields (seed and lint) since it had the highest values in the first pick. G.85 and G.86 were more larger than other genotypes with respect to lint percentage and fiber length, respectively in the two picks.

\subsection{Combined latin square design}

Two analyses of combined latin square to estimate the environments variance in the first and the second pick by two ways direct and indirect.

\subsubsection{First analysis (direct) two picks}

The analysis of variance of the two combined (one the first pick and the other the second) 
Table (3): Means of yield, its components and fiber for simple latin square.

\begin{tabular}{|c|c|c|c|c|c|c|}
\hline \multicolumn{7}{|c|}{ First pick } \\
\hline Traits & \multicolumn{2}{|c|}{ S. C. Y. (k/fed) } & \multicolumn{2}{|c|}{ L. C. Y. $(\mathrm{k} / \mathrm{fed})$} & \multicolumn{2}{|c|}{ B. W. (gm) } \\
\hline Genotypes & Gharbia & Mounofia & Gharbia & Mounofia & Gharbia & Mounofia \\
\hline G.85 & 6.95 & 8.17 & 8.58 & 10.28 & 2.82 & 2.73 \\
\hline G.86 & 6.32 & 6.22 & 7.75 & 7.81 & 3.19 & 2.90 \\
\hline G.89 & 6.31 & 6.91 & 7.31 & 8.32 & 3.17 & 2.69 \\
\hline G.89 x G.86 & 7.71 & 10.53 & 9.12 & 12.73 & 3.07 & 3.14 \\
\hline G.89 x Pima S-6 & 8.78 & 11.14 & 10.58 & 13.66 & 2.71 & 2.76 \\
\hline L.S.D. & 1.08 & 0.87 & 1.29 & 1.08 & 0.26 & 0.17 \\
\hline Genotypes & \multicolumn{2}{|c|}{ L.P. $(\%)$} & \multicolumn{2}{|c|}{ S. I. (gm) } & \multicolumn{2}{|c|}{ L. I. (gm) } \\
\hline G.85 & 39.33 & 39.94 & 9.74 & 8.92 & 6.31 & 5.93 \\
\hline G.86 & 39.05 & 39.75 & 10.58 & 9.71 & 6.78 & 6.43 \\
\hline G.89 & 36.85 & 38.31 & 10.18 & 8.78 & 5.93 & 5.45 \\
\hline G.89 x G.86 & 37.72 & 38.39 & 10.69 & 10.70 & 6.47 & 6.67 \\
\hline G.89 x Pima S-6 & 38.36 & 38.93 & 10.08 & 10.38 & 6.26 & 6.62 \\
\hline L.S.D. & 0.97 & 1.10 & ---- & $\mathbf{0 . 8 2}$ & 0.37 & 0.55 \\
\hline Genotypes & \multicolumn{2}{|c|}{ F.L. $(\mathbf{m m})$} & \multicolumn{2}{|c|}{ Mic. } & & \\
\hline G.85 & 29.74 & 31.04 & 4.08 & 4.36 & & \\
\hline G.86 & 32.64 & 33.30 & 4.48 & 4.60 & & \\
\hline G.89 & 31.88 & 32.44 & 4.52 & 4.64 & & \\
\hline G.89 x G.86 & 32.38 & 31.72 & 4.60 & 4.88 & & \\
\hline G.89 x Pima S-6 & 29.88 & 30.80 & 4.24 & 4.24 & & \\
\hline L.S.D. & 0.89 & 0.75 & $\mathbf{0 . 3 2}$ & 0.36 & & \\
\hline \multicolumn{7}{|c|}{ Second pick } \\
\hline Traits & \multicolumn{2}{|c|}{ S. C. Y. (k/fed) } & \multicolumn{2}{|c|}{ L. C. Y. $(k / f e d)$} & \multicolumn{2}{|c|}{ B. W. (gm) } \\
\hline Genotypes & Gharbia & Mounofia & Gharbia & Mounofia & Gharbia & Mounofia \\
\hline G.85 & 2.50 & 2.10 & 3.15 & 2.74 & 2.38 & 2.29 \\
\hline G.86 & 2.59 & 2.24 & 3.38 & 2.36 & 2.64 & 2.34 \\
\hline G.89 & 2.81 & 2.32 & 3.41 & 2.90 & 2.52 & 2.37 \\
\hline G.89 x G.86 & 2.04 & 1.69 & 2.53 & 1.92 & 2.51 & 2.38 \\
\hline G.89 x Pima S-6 & 1.88 & 1.62 & 2.35 & 2.11 & 2.28 & 2.23 \\
\hline L.S.D. & 0.26 & $\mathbf{0 . 5 2}$ & $\mathbf{0 . 3 2}$ & 0.60 & 0.18 & $-\cdots$ \\
\hline Genotypes & \multicolumn{2}{|c|}{ L. P. (\%) } & \multicolumn{2}{|c|}{ S. I. (gm) } & \multicolumn{2}{|c|}{ L. I. (gm) } \\
\hline G.85 & 40.18 & 41.54 & 7.43 & 6.90 & 4.99 & 4.90 \\
\hline G.86 & 41.22 & 41.14 & 8.78 & 7.63 & 6.16 & 5.34 \\
\hline G.89 & 38.60 & 39.66 & 8.47 & 6.51 & 5.33 & 4.28 \\
\hline G.89 x G.86 & 39.57 & 39.67 & 8.76 & 8.43 & 5.74 & 5.54 \\
\hline G.89 x Pima S-6 & 39.76 & 41.10 & 7.63 & 8.08 & 5.03 & 5.65 \\
\hline L.S.D. & 1.06 & 0.87 & 0.65 & 0.86 & 0.44 & 0.59 \\
\hline Genotypes & \multicolumn{2}{|c|}{ F.L. $(\mathbf{m m})$} & \multicolumn{2}{|c|}{ Mic. } & & \\
\hline G.85 & 28.18 & 28.60 & 3.60 & 3.32 & & \\
\hline G.86 & 31.46 & 31.72 & 4.08 & 3.56 & & \\
\hline G.89 & 30.52 & 30.30 & 4.20 & 3.64 & & \\
\hline G.89 x G.86 & 31.00 & 29.94 & 4.02 & 3.78 & & \\
\hline G.89 x Pima S-6 & 28.58 & 28.86 & 3.76 & 3.60 & & \\
\hline L.S.D. & 1.33 & 0.94 & 0.30 & ---- & & \\
\hline
\end{tabular}

revealed the presence of significance environments, genotypes and the interaction between them, (Table 4). In the first and the second pick, significant variation due to environments was detected for yields (seed and lint), yield components and fiber properties except lint index for the first pick and fiber length for the second pick. Significant variation due to genotypes was observed for all traits in the first and the second pick. Significant variation due to genotypes $\mathrm{x}$ environments was recorded for yields (seed and lint), boll weight and fiber length with respect to the first pick, seed index and lint index with respect to the second pick.

\subsubsection{Second analysis (indirect) four picks}

The analysis of variance of combined (four picks) revealed the presence of significance picks, partitioning of picks, genotypes and the interaction between genotypes x picks (Table 5). Significant variation due to picks and partitioning of them were detected for yields (seed and lint), yield components and fiber properties except lint index for first pick and fiber length for second pick. Significant variations due to genotypes and genotypes $\mathrm{x}$ picks were observed for all traits except lint percentage, fiber length and micronaire reading.

\subsubsection{Comparison between (direct and indirect)}


Direct depends on two analysis of combined to estimate environments variance in the first pick and the second pick. Indirect surpassed direct due to it needed one analysis of combined to estimate environments variance in the first pick and the second pick. Although direct and indirect calculated the same values of environments variance in the first pick and the second picks but they exhibited different results of significant variation due to different values of tablulated $\mathrm{F}$ of them, which depends on degrees of freedom of error and different experimental error.

Table (4): Mean squares of yield, its components and fiber properties for combined latin square (two picks).

\begin{tabular}{|c|c|c|c|c|c|c|c|}
\hline \multicolumn{2}{|l|}{ Traits } & \multicolumn{2}{|c|}{ S. C. Y. (k/fed) } & \multicolumn{2}{|c|}{ L. C. Y. (k/fed) } & \multicolumn{2}{|c|}{ B. W. (gm) } \\
\hline Source of variation & d.f. & First & Second & First & Second & First & Second \\
\hline Environments (E) & 1 & $23.82 * *$ & $1.71 * *$ & $44.56 * *$ & $3.94 * *$ & $0.272 * *$ & $0.259 * *$ \\
\hline Rows within (E) & 8 & $2.67 * *$ & $1.22 * *$ & $4.22 * *$ & $2.01 * *$ & 0.020 & $0.045^{*}$ \\
\hline Columns within (E) & 8 & $2.10 * *$ & $0.498 * *$ & $3.33 * *$ & $0.980 * *$ & $\mathbf{0 . 0 5 0}$ & 0.029 \\
\hline Genotypes within (E) & 8 & $14.54 * *$ & $0.639 * *$ & $20.96 * *$ & $1.04 * *$ & $0.201 * *$ & $0.059 *$ \\
\hline Genotypes (G) & 4 & $25.42 * *$ & $1.26 * *$ & $36.58 * *$ & $1.87 * *$ & $0.268 * *$ & $0.097 * *$ \\
\hline $\mathbf{G} \times \mathbf{E}$ & 4 & $3.66 * *$ & 0.019 & $5.34 * *$ & 0.218 & $0.135 * *$ & 0.022 \\
\hline Experimental error & 24 & 0.507 & 0.089 & 0.746 & 0.123 & 0.026 & 0.018 \\
\hline Source of variation & d.f. & \multicolumn{2}{|c|}{ L. P. $(\%)$} & \multicolumn{2}{|c|}{ S. I. (gm) } & \multicolumn{2}{|c|}{ L. I. (gm) } \\
\hline Environments (E) & 1 & $8.09 * *$ & $7.11 * *$ & $3.83 * *$ & $6.24 * *$ & 0.220 & $1.18 * *$ \\
\hline Rows within (E) & 8 & 0.261 & 0.453 & 0.825 & $0.794 *$ & $0.454 * *$ & $0.499 * *$ \\
\hline Columns within (E) & 8 & 0.251 & 0.412 & 0.200 & 0.320 & 0.106 & 0.154 \\
\hline Genotypes within (E) & & $3.97 * *$ & $4.26 * *$ & $2.20 * *$ & $2.65 * *$ & $0.911 * *$ & $1.40 * *$ \\
\hline Genotypes (G) & 4 & $7.59 * *$ & $7.30 * *$ & $3.19 * *$ & $3.22 * *$ & $1.46 * *$ & $1.74 * *$ \\
\hline $\mathbf{G} \times \mathbf{E}$ & 4 & 0.349 & 1.22 & 1.21 & $2.07 * *$ & 0.363 & $1.07 * *$ \\
\hline Experimental error & 24 & 0.566 & 0.492 & 0.371 & 0.303 & 0.116 & 0.144 \\
\hline Source of variation & d.f. & \multicolumn{2}{|c|}{ F.L. $(\mathbf{m m})$} & \multicolumn{2}{|c|}{ Mic. } & & \\
\hline Environments (E) & 1 & $3.86^{* *}$ & 0.370 & $0.320 *$ & $1.55 * *$ & & \\
\hline Rows within (E) & 8 & 0.369 & 1.26 & 0.046 & 0.062 & & \\
\hline Columns within $(E)$ & 8 & 0.255 & 0.421 & 0.034 & 0.051 & & \\
\hline Genotypes within (E) & 8 & $7.48 * *$ & $9.22 * *$ & $0.275 * *$ & $0.221 * *$ & & \\
\hline Genotypes (G) & 4 & $13.61 * *$ & $17.26 * *$ & $0.513 * *$ & $0.363 * *$ & & \\
\hline $\mathbf{G} \times \mathbf{E}$ & 4 & $1.36 *$ & 1.17 & $\mathbf{0 . 0 3 7}$ & 0.080 & & \\
\hline Experimental error & 24 & 0.354 & 0.751 & 0.061 & 0.054 & & \\
\hline
\end{tabular}

Table (5): Mean squares of yield, its components and fiber properties for combined latin square (four picks).

\begin{tabular}{|c|c|c|c|c|c|}
\hline \multicolumn{2}{|l|}{ Traits } & \multirow{2}{*}{$\begin{array}{c}\text { S. C. Y. } \\
\text { (k/fed) }\end{array}$} & \multirow{2}{*}{$\begin{array}{c}\text { L. C. Y. } \\
\text { (k/fed) }\end{array}$} & \multirow{2}{*}{$\begin{array}{l}\text { B. W. } \\
\text { (gm) }\end{array}$} & \multirow{2}{*}{$\begin{array}{c}\text { L.P. } \\
(\%)\end{array}$} \\
\hline Source of variation & d.f. & & & & \\
\hline Picks & 3 & $281.56 * *$ & $416.42 * *$ & $2.47 * *$ & $25.88 * *$ \\
\hline First (different environments) & 1 & $23.82 * *$ & $44.56 * *$ & $0.272 * *$ & $8.09 * *$ \\
\hline Second (different environments) & 1 & $1.71 *$ & $3.94 * *$ & $0.259 * *$ & $7.11 * *$ \\
\hline First vs. Second & 1 & $819.16 * *$ & $1200.76 * *$ & $6.88 * *$ & $62.43 * *$ \\
\hline Rows within picks & 16 & $1.94 * *$ & $3.11 * *$ & $\mathbf{0 . 0 3 2}$ & 0.357 \\
\hline Columns within picks & 16 & $1.30 * *$ & $2.16 * *$ & $\mathbf{0 . 0 3 9}$ & 0.332 \\
\hline Genotypes within picks & 16 & $7.59 * *$ & $11.00 * *$ & $0.130 * *$ & $4.11 * *$ \\
\hline Genotypes & 4 & $7.86 * *$ & $11.75 * *$ & $0.327 * *$ & $14.62 * *$ \\
\hline Genotypes $x$ picks & 12 & $7.50 * *$ & $10.75 * *$ & $0.065 * *$ & 0.611 \\
\hline Experimental error & 48 & 0.298 & 0.434 & 0.022 & 0.529 \\
\hline Source of variation & d.f. & S. I. (gm) & L. I. (gm) & F.L. (mm) & Mic. \\
\hline Picks & 3 & $40.54 * *$ & $8.62 * *$ & $26.07 * *$ & $4.80 * *$ \\
\hline First (different environments) & 1 & $3.83 * *$ & 0.220 & $3.86 * *$ & $0.320 *$ \\
\hline Second (different environments) & 1 & $6.24 * *$ & $1.18 * *$ & 0.370 & $1.55 * *$ \\
\hline First vs. Second & 1 & $111.54 * *$ & $24.46 * *$ & $73.96 * *$ & $12.53 * *$ \\
\hline Rows within picks & 16 & $0.809 *$ & $0.477 * *$ & 0.814 & 0.054 \\
\hline Columns within picks & 16 & 0.260 & 0.130 & 0.338 & 0.042 \\
\hline Genotypes within picks & 16 & $2.42 * *$ & $1.16 * *$ & $8.35 * *$ & $0.248 * *$ \\
\hline Genotypes & 4 & $6.28 * *$ & $3.10 * *$ & $30.48 * *$ & $0.820 * *$ \\
\hline Genotypes $x$ picks & 12 & $1.14 * *$ & $0.511 * *$ & 0.975 & 0.057 \\
\hline Experimental error & 48 & 0.337 & 0.130 & 0.553 & 0.058 \\
\hline
\end{tabular}


Table (6): Means genotypes (two picks) or means genotypes x picks (four picks) for combined latin square.

\begin{tabular}{|c|c|c|c|c|c|c|}
\hline Traits & \multicolumn{2}{|c|}{ S. C. Y. (k/fed) } & \multicolumn{2}{|c|}{ L. C. Y. (k/fed) } & \multicolumn{2}{|c|}{ B. W. (gm) } \\
\hline Genotypes & First & Second & First & Second & First & Second \\
\hline G.85 & 7.56 & 2.30 & 9.43 & 2.95 & 2.77 & 2.33 \\
\hline G.86 & 6.27 & 2.41 & 7.78 & 2.87 & 3.05 & 2.49 \\
\hline G.89 & 6.61 & 2.66 & 7.82 & 3.16 & 2.93 & 2.45 \\
\hline G.89 x G.86 & 9.12 & 1.86 & 10.93 & 2.22 & 3.11 & 2.45 \\
\hline G.89 x Pima S-6 & 10.00 & 1.75 & 12.12 & 2.23 & 2.73 & 2.26 \\
\hline L.S.D. & 0.66 & 0.27 & 0.80 & 0.32 & 0.15 & 0.12 \\
\hline L.S.D. & \multicolumn{2}{|c|}{0.35} & \multicolumn{2}{|c|}{0.42} & \multicolumn{2}{|c|}{0.09} \\
\hline Genotypes & \multicolumn{2}{|c|}{ L. P. (\%) } & \multicolumn{2}{|c|}{ S. I. (gm) } & \multicolumn{2}{|c|}{ L. I. (gm) } \\
\hline G.85 & 39.64 & 40.86 & 9.33 & 7.16 & 6.12 & 4.95 \\
\hline G.86 & 39.40 & 41.18 & 10.15 & 8.21 & 6.60 & 5.75 \\
\hline G.89 & 37.58 & 39.13 & 9.48 & 7.49 & 5.69 & 4.80 \\
\hline G.89 x G.86 & 38.06 & 39.62 & 10.69 & 8.60 & 6.57 & 5.64 \\
\hline G.89 x Pima S-6 & 38.64 & 40.43 & 10.23 & 7.86 & 6.44 & 5.34 \\
\hline L.S.D. & 0.69 & 0.65 & 0.56 & 0.51 & 0.31 & 0.35 \\
\hline L.S.D. & \multicolumn{2}{|c|}{----} & \multicolumn{2}{|c|}{0.37} & \multicolumn{2}{|c|}{0.23} \\
\hline Genotypes & \multicolumn{2}{|c|}{ F.L. (mm) } & \multicolumn{2}{|c|}{ Mic. } & & \\
\hline G.85 & 30.39 & 28.39 & 4.22 & 3.46 & & \\
\hline G.86 & 32.97 & 31.59 & 4.54 & 3.82 & & \\
\hline G.89 & 32.16 & 30.14 & 4.58 & 3.92 & & \\
\hline G.89 x G.86 & 32.05 & 30.47 & 4.74 & 3.90 & & \\
\hline G.89 x Pima S-6 & 30.34 & 28.72 & 4.24 & 3.68 & & \\
\hline L.S.D. & 0.55 & 0.80 & 0.23 & 0.21 & & \\
\hline L.S.D. & & & & & & \\
\hline
\end{tabular}

\subsection{Multiple regression}

\subsubsection{Simple latin square}

In Gharbia, in the second pick, when considered the effect of boll weight and then the additional effect of seed index on seed cotton yield exhibited effect of boll weight was significant with respect to G.86. On contrast, when considered the effect of seed index and then the additional effect boll weight on seed cotton yield exhibited effect of boll weight was significant with respect to G.89, (Table 7).

In Gharbia, in the first pick, when considered the effect of boll weight and then the additional effect of lint percentage on lint cotton yield exhibited effect of lint percentage was significant with respect to G.89 x G.86. On contrast, when considered the effect of lint percentage and then the additional effect boll weight on lint cotton yield exhibited effect of boll weight was significant with respect to G.89 x G.86, (Table 8).
In Gharbia, in the second pick, when considered the effect of boll weight and then the additional effect of lint percentage on lint cotton yield exhibited effect of boll weight was significant with respect to G.86, (Table 8).

\subsubsection{Combined latin square (two picks)}

In the second pick, when considered the effect of boll weight and then the additional effect of seed index on seed cotton yield exhibited effect of boll weight was significant with respect to G. $89 \mathrm{x}$ Pima S-6. On contrast, when considered the effect of seed index and then the additional effect boll weight on seed cotton yield exhibited effect of boll weight was significant with respect to G. $89 \mathrm{x}$ Pima S-6, (Table 9).

In the second pick, when considered the effect of lint percentage and then the additional effect boll weight on lint cotton yield exhibited effect of boll weight was significant with respect to $\mathrm{G} .89 \mathrm{x}$ Pima S-6, (Table 10). 
Table (7): Mean square of multiple regression of seed cotton yield (Y), boll weight $\left(\mathrm{X}_{1}\right)$ and seed index $\left(\mathbf{X}_{2}\right)$ for simple latin square.

\begin{tabular}{|c|c|c|c|c|c|c|}
\hline \multicolumn{7}{|c|}{ Gharbia 2005 Season } \\
\hline \multicolumn{7}{|c|}{$\begin{array}{l}\text { First pick } \\
\end{array}$} \\
\hline Genotypes & & G.85 & G.86 & G.89 & $89 \times 86$ & 89xPima \\
\hline Source of variation & d.f. & & & & & S-6 \\
\hline Total & 4 & & & & & \\
\hline Regression due to (B.W.) & 1 & 0.113 & 0.043 & 1.58 & 0.002 & 2.49 \\
\hline Deviation from simple regression & 3 & 2.94 & 1.21 & 1.61 & 1.22 & 0.687 \\
\hline Additional regression due to (S.I.) & 1 & 4.75 & 2.88 & 3.55 & 0.155 & 0.147 \\
\hline Deviation from multiple regression & 2 & 2.03 & 0.380 & 0.650 & 1.75 & 0.957 \\
\hline Total & 4 & & & & & \\
\hline Regression due to (S.I.) & 1 & 2.37 & 1.35 & 4.29 & 0.057 & 0.856 \\
\hline Deviation from simple regression & 3 & 2.18 & 0.773 & 0.710 & 1.20 & 1.23 \\
\hline Additional regression due to (B.W.) & 1 & 2.48 & 1.57 & $\mathbf{0 . 8 3 9}$ & 0.099 & 1.79 \\
\hline Deviation from multiple regression & 2 & 2.04 & $\mathbf{0 . 3 7 5}$ & 0.651 & 1.75 & 0.950 \\
\hline \multicolumn{7}{|c|}{ Second pick } \\
\hline Total & 4 & & & & & \\
\hline Regression due to (B.W.) & 1 & 0.543 & $0.719 *$ & 1.19 & 0.025 & 2.40 \\
\hline Deviation from simple regression & 3 & 0.279 & $\mathbf{0 . 0 5 2}$ & 0.407 & 0.318 & 0.347 \\
\hline Additional regression due to (S.I.) & 1 & 0.121 & $\mathbf{0 . 0 5 3}$ & 1.10 & 0.851 & 0.413 \\
\hline Deviation from multiple regression & 2 & 0.358 & $\mathbf{0 . 0 5 2}$ & 0.060 & $\mathbf{0 . 0 5 2}$ & 0.314 \\
\hline Total & 4 & & & & & \\
\hline Regression due to (S.I.) & 1 & 0.038 & 0.479 & 0.093 & 0.205 & 2.49 \\
\hline Deviation from simple regression & 3 & 0.447 & 0.132 & 0.772 & 0.258 & 0.317 \\
\hline Additional regression due to (B.W.) & 1 & 0.627 & 0.292 & $2.20 *$ & 0.672 & 0.322 \\
\hline Deviation from multiple regression & 2 & $\mathbf{0 . 3 5 7}$ & $\mathbf{0 . 0 5 2}$ & 0.060 & 0.052 & 0.314 \\
\hline \multicolumn{7}{|c|}{ Mounofia 2006 Season } \\
\hline \multicolumn{7}{|c|}{ First pick } \\
\hline Total & 4 & & & & & \\
\hline Regression due to (B.W.) & 1 & 0.015 & 0.004 & 5.33 & 0.374 & 0.006 \\
\hline Deviation from simple regression & 3 & 0.462 & 2.29 & 1.04 & 1.03 & 0.971 \\
\hline Additional regression due to (S.I.) & 1 & 0.493 & 2.46 & 0.163 & 0.810 & 0.543 \\
\hline Deviation from multiple regression & 2 & 0.449 & 2.19 & 1.48 & 1.14 & 1.18 \\
\hline Total & 4 & & & & & \\
\hline Regression due to (S.I.) & 1 & 0.210 & 2.45 & 3.75 & 0.974 & 0.404 \\
\hline Deviation from simple regression & 3 & 0.397 & 1.47 & 1.57 & 0.829 & 1.84 \\
\hline Additional regression due to (B.W.) & 1 & 0.297 & 0.015 & 1.74 & 0.211 & 0.144 \\
\hline Deviation from multiple regression & 2 & 0.447 & 2.20 & 1.48 & 1.14 & 2.69 \\
\hline \multicolumn{7}{|c|}{ Second pick } \\
\hline Total & 4 & & & & & \\
\hline Regression due to (B.W.) & 1 & 0.022 & 0.001 & 0.200 & 0.007 & 0.597 \\
\hline Deviation from simple regression & 3 & 0.252 & 0.670 & 0.943 & 0.401 & 0.468 \\
\hline Additional regression due to (S.I.) & $\mathbf{1}$ & 0.059 & 0.005 & 1.22 & 0.056 & $\mathbf{0 . 0 3 2}$ \\
\hline Deviation from multiple regression & 2 & 0.349 & 1.00 & 0.805 & $\mathbf{0 . 5 7 2}$ & 0.684 \\
\hline Total & 4 & & & & & \\
\hline Regression due to (S.I.) & $\mathbf{1}$ & 0.079 & 0.005 & 0.707 & 0.024 & 0.084 \\
\hline Deviation from simple regression & 3 & 0.233 & 0.668 & 0.774 & 0.395 & 0.639 \\
\hline Additional regression due to (B.W.) & 1 & 0.002 & 0.002 & 0.711 & 0.040 & 0.546 \\
\hline Deviation from multiple regression & 2 & 0.349 & 1.00 & 0.804 & $\mathbf{0 . 5 7 5}$ & 0.687 \\
\hline
\end{tabular}


Table (8): Mean square of multiple regression of lint cotton yield (Y), boll weight $\left(\mathrm{X}_{1}\right)$ and lint percentage $\left(\mathbf{X}_{2}\right)$ for simple latin square.

\begin{tabular}{|c|c|c|c|c|c|c|}
\hline \multicolumn{7}{|c|}{ Gharbia 2005 Season } \\
\hline \multicolumn{7}{|c|}{ First pick } \\
\hline Genotypes & & G.85 & G.86 & G.89 & $89 \times 86$ & 89xPima \\
\hline Source of variation & d.f. & & & & & S-6 \\
\hline Total & 4 & & & & & \\
\hline Regression due to (B.W.) & 1 & 0.163 & 0.168 & 2.23 & 0.092 & 4.20 \\
\hline Deviation from simple regression & 3 & 4.45 & 1.92 & 2.43 & 1.38 & 1.12 \\
\hline Additional regression due to (L.P.) & 1 & 0.262 & 0.694 & 3.53 & 4.03* & 0.569 \\
\hline Deviation from multiple regression & 2 & 6.54 & 2.53 & 1.88 & 0.050 & 1.40 \\
\hline Total & 4 & & & & & \\
\hline Regression due to (L.P.) & 1 & 0.338 & 0.857 & 5.01 & 1.41 & 3.11 \\
\hline Deviation from simple regression & 3 & 4.39 & 1.69 & 1.50 & 0.937 & 1.48 \\
\hline Additional regression due to (B.W.) & 1 & 0.087 & 0.006 & 0.758 & $2.70 *$ & 1.66 \\
\hline Deviation from multiple regression & 2 & 6.54 & 2.53 & 1.87 & 0.055 & 1.40 \\
\hline \multicolumn{7}{|c|}{ Second pick } \\
\hline Total & 4 & & & & & \\
\hline Regression due to (B.W.) & 1 & 0.995 & $1.20 *$ & 1.83 & 0.083 & 3.76 \\
\hline Deviation from simple regression & 3 & 0.412 & 0.117 & 0.597 & 0.499 & 0.523 \\
\hline Additional regression due to (L.P.) & 1 & 0.906 & 0.187 & 0.006 & 0.072 & 0.075 \\
\hline Deviation from multiple regression & 2 & 0.167 & 0.082 & 0.892 & 0.714 & 0.748 \\
\hline Total & 4 & & & & & \\
\hline Regression due to (L.P.) & 1 & 0.042 & 0.144 & 0.084 & 0.101 & 0.328 \\
\hline Deviation from simple regression & 3 & 0.729 & 0.469 & 1.18 & 0.493 & 1.67 \\
\hline Additional regression due to (B.W.) & 1 & 1.86 & 1.25 & 1.76 & 0.054 & 3.51 \\
\hline Deviation from multiple regression & 2 & 0.165 & 0.080 & 0.890 & 0.713 & 0.745 \\
\hline \multicolumn{7}{|c|}{ Mounofia 2006 Season } \\
\hline \multicolumn{7}{|c|}{ First pick } \\
\hline Total & 4 & & & & & \\
\hline Regression due to (B.W.) & 1 & 0.017 & 6.11 & 6.95 & 0.887 & 0.044 \\
\hline Deviation from simple regression & 3 & 0.791 & 2.42 & 1.42 & 1.63 & 1.59 \\
\hline Additional regression due to (L.P.) & 1 & 0.408 & 2.71 & 1.31 & 3.70 & 0.422 \\
\hline Deviation from multiple regression & 2 & 0.981 & 2.28 & 1.48 & 0.600 & 2.18 \\
\hline Total & 4 & & & & & \\
\hline Regression due to (L.P.) & 1 & 0.116 & 5.27 & 7.57 & 2.27 & 0.382 \\
\hline Deviation from simple regression & 3 & 0.758 & 2.70 & 1.21 & 1.17 & 1.48 \\
\hline Additional regression due to (B.W.) & 1 & 0.310 & 3.55 & 0.690 & 2.31 & 0.084 \\
\hline Deviation from multiple regression & 2 & 0.980 & 2.28 & 1.48 & 0.605 & 2.18 \\
\hline \multicolumn{7}{|c|}{ Second pick } \\
\hline Total & 4 & & & & & \\
\hline Regression due to (B.W.) & $\mathbf{1}$ & 0.055 & 0.011 & 0.333 & 0.013 & 1.00 \\
\hline Deviation from simple regression & 3 & 0.432 & 1.17 & 1.53 & 0.626 & 0.847 \\
\hline Additional regression due to (L.P.) & 1 & 0.030 & 0.121 & 0.518 & 0.032 & 1.58 \\
\hline Deviation from multiple regression & 2 & 0.635 & 1.69 & 2.03 & 0.924 & 0.480 \\
\hline Total & 4 & & & & & \\
\hline Regression due to (L.P.) & 1 & 0.017 & 0.104 & 0.548 & $\mathbf{0 . 0 3 1}$ & 1.06 \\
\hline Deviation from simple regression & 3 & 0.444 & 1.14 & 1.45 & 0.620 & 0.827 \\
\hline Additional regression due to (B.W.) & 1 & 0.068 & 0.028 & 0.302 & 0.014 & 1.53 \\
\hline Deviation from multiple regression & 2 & 0.631 & 1.69 & 2.03 & 0.923 & 0.475 \\
\hline
\end{tabular}


Table (9): Mean square of multiple regression of seed cotton yield (Y), boll weight $\left(X_{1}\right)$ and seed index $\left(X_{2}\right)$ for combined latin square (two picks).

\begin{tabular}{|c|c|c|c|c|c|c|}
\hline \multicolumn{7}{|c|}{ First pick } \\
\hline Genotypes & & G.85 & G.86 & G.89 & $89 \times 86$ & 89x Pima \\
\hline Source of variation & d.f. & & & & & S-6 \\
\hline Total & 9 & & & & & \\
\hline Regression due to (B.W.) & 1 & 1.08 & 0.841 & 0.182 & 0.001 & 0.018 \\
\hline Deviation from simple regression & 8 & 1.62 & 1.20 & 1.95 & 3.37 & 2.68 \\
\hline Additional regression due to (S.I.) & 1 & 0.601 & 1.83 & 0.628 & 0.491 & 0.499 \\
\hline Deviation from multiple regression & 7 & 1.76 & 1.12 & 2.14 & 3.78 & 2.99 \\
\hline Total & 9 & & & & & \\
\hline Regression due to (S.I.) & 1 & 0.059 & 0.157 & 0.147 & 0.485 & 0.420 \\
\hline Deviation from simple regression & 8 & 1.75 & 1.29 & 1.96 & 3.31 & 2.63 \\
\hline Additional regression due to (B.W.) & 1 & 1.63 & 2.51 & 0.664 & 0.007 & 0.097 \\
\hline Deviation from multiple regression & 7 & 1.76 & 1.12 & 2.14 & 3.78 & 2.99 \\
\hline \multicolumn{7}{|c|}{ Second pick } \\
\hline Total & 9 & & & & & \\
\hline Regression due to (B.W.) & 1 & 0.792 & 1.62 & 1.94 & 0.002 & $2.98 *$ \\
\hline Deviation from simple regression & 8 & 0.221 & 0.341 & 0.514 & 0.311 & 0.310 \\
\hline Additional regression due to (S.I.) & 1 & 0.021 & 0.224 & 0.262 & 0.171 & 0.221 \\
\hline Deviation from multiple regression & 7 & 0.250 & 0.358 & 0.550 & 0.331 & 0.323 \\
\hline Total & 9 & & & & & \\
\hline Regression due to (S.I.) & 1 & 0.125 & 1.21 & 0.012 & 0.100 & 1.08 \\
\hline Deviation from simple regression & 8 & 0.304 & 0.393 & 0.755 & 0.299 & 0.548 \\
\hline Additional regression due to (B.W.) & 1 & 0.687 & 0.635 & 2.19 & 0.072 & $2.16 *$ \\
\hline Deviation from multiple regression & 7 & 0.250 & 0.358 & $\mathbf{0 . 5 5 0}$ & 0.331 & 0.317 \\
\hline
\end{tabular}

Table (10): Mean square of multiple regression of lint cotton yield (Y), boll weight $\left(\mathrm{X}_{1}\right)$ and lint percentage $\left(\mathrm{X}_{2}\right)$ for combined latin square (two picks).

\begin{tabular}{|c|c|c|c|c|c|c|}
\hline \multicolumn{7}{|c|}{ First pick } \\
\hline Genotypes & & G.85 & G.86 & G.89 & $89 \times 86$ & 89x Pima \\
\hline Source of variation & d.f. & & & & & S-6 \\
\hline Total & 9 & & & & & \\
\hline Regression due to (B.W.) & 1 & 1.58 & 2.34 & 0.017 & 0.014 & 0.189 \\
\hline Deviation from simple regression & 8 & 2.68 & 2.12 & 2.91 & 5.33 & 4.47 \\
\hline Additional regression due to (L.P.) & 1 & 3.21 & 3.29 & 3.92 & 9.15 & 14.84 \\
\hline Deviation from multiple regression & 7 & 2.61 & 1.95 & 2.76 & 4.78 & 2.99 \\
\hline Total & 9 & & & & & \\
\hline Regression due to (L.P.) & 1 & 2.41 & 5.42 & 2.09 & 8.59 & 14.14 \\
\hline Deviation from simple regression & 8 & 2.58 & 1.74 & 2.65 & 4.26 & 2.73 \\
\hline Additional regression due to (B.W.) & 1 & 2.38 & 0.219 & 0.184 & 0.579 & 0.893 \\
\hline Deviation from multiple regression & 7 & 2.60 & 1.95 & 2.76 & 4.78 & 2.99 \\
\hline \multicolumn{7}{|c|}{ Second pick } \\
\hline Total & 9 & & & & & \\
\hline Regression due to (B.W.) & 1 & 1.34 & 2.95 & 2.64 & 0.007 & 0.466 \\
\hline Deviation from simple regression & 8 & 0.334 & 0.593 & 0.819 & 0.488 & 0.526 \\
\hline Additional regression due to (L.P.) & 1 & 0.367 & 0.002 & 0.361 & 0.085 & 0.469 \\
\hline Deviation from multiple regression & 7 & 0.329 & 0.677 & 0.884 & 0.545 & 0.534 \\
\hline Total & 9 & & & & & \\
\hline Regression due to (L.P.) & 1 & 0.188 & 0.833 & 0.004 & 0.082 & 0.530 \\
\hline Deviation from simple regression & 8 & 0.478 & 0.857 & 1.15 & 0.479 & 1.04 \\
\hline Additional regression due to $(\mathrm{B} . \mathrm{W})$. & 1 & 1.52 & 2.13 & 3.01 & 0.009 & $4.60 *$ \\
\hline Deviation from multiple regression & 7 & 0.329 & 0.676 & 0.883 & 0.546 & 0.534 \\
\hline
\end{tabular}




\section{REFERENCES}

Abo Tour H. B., Seyam S. M. and Abd ElRahman L. M. (1996). Analytical study on the economic characters of new and commercial Egyptian cotton. Egypt. J. Agric. Res., 74 (3) : 781-791.

Badr S.S. and El Sayed S. A. (2004). Evaluation of some long staple Egyptian cotton genotypes for yield, seed quality and seed viability characters. J. Agric. Res. Tanta Univ., 30 (2) : 304-326.

Bailey N. T. (1994). Statistical Methods in Biology. Third Edit. Cambridge University Press, Cambridge, England.

Cochran W.G. and Cox G. M. (1950). Experimental Designs. John Wiley and Sons, New York., U.S.A.

El Shaer M. H., Shabana R., El Sheikh A. H. and Abd El Rahman L. M. (1984). Effect of picking period and boll position on fiber quality characters in some Egyptian and Upland cottons. Agric. Res. Rev., Egypt, 62 : $165-174$.
Federer W. T. (1955). Experimental Design. Theory and Application. The Macmillan Company New York, U.S.A.

Gomez K. A. and Gomez A. A. (1984). Statistical Procedures for Agricultural Research. Wiley and Sons, New York, U.S.A.

Idris H.A. and Abd El-Rahman L. M. (2006). Estimation and classification of variance between the first and the second picks in some Egyptian cotton genotypes by using latin square design and cluster analysis. Bull. Fac. Agric. Cairo Univ., 57 (2): 277-294.

Little T.M. and Hills F. J. (1978). Agricultural Experimentation Design and Analysis. John Wiley and Sons, New York., U.S.A.

Roger G. P. (1994). Agricultural Field Experiments Design and Analysis. Marcel Dekker, Inc. New York, U.S.A.

Sing P. and Narayanan S. S. (2000). Biometrical Techniques in Plant Breeding. Second Edit., Ludhiana, New Delhi. India.

Steel R. G. and Torrie J. H. (1980). Principles and Procedures of Statistics. Second Edit., McGraw. Hill. Book Co. New York, U.S.A.

$$
\begin{aligned}
& \text { تقييم لبعض التراكيب الوراثية من القطن المصري فى الجنية الأولى والثانية }
\end{aligned}
$$

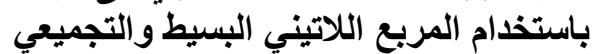

$$
\begin{aligned}
& \text { حاتم أحمد إدريس اعزئ } \\
& \text { معهد بحوث القطن - مركز البحوث الزر اعية ـ الجيزة ـ مصر. } \\
& \text { ملخص الإن الزر }
\end{aligned}
$$

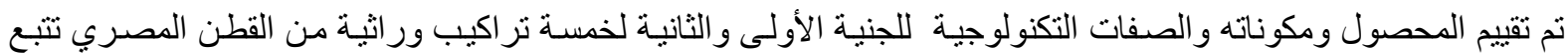

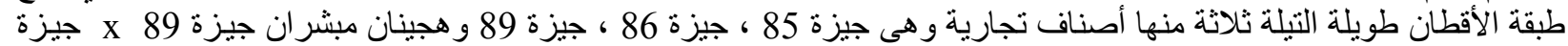

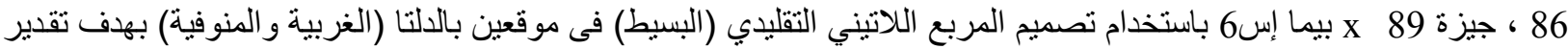

$$
\begin{aligned}
& \text { تباين التز اكيب الور اثية في كل جنية. }
\end{aligned}
$$

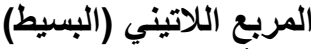

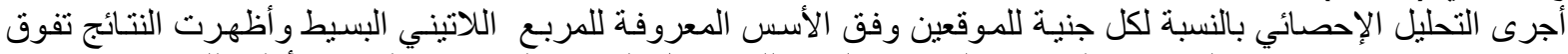

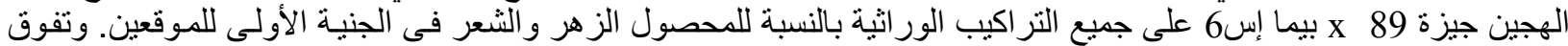

$$
\begin{aligned}
& \text { الصنف جيزة } 86 \text { على جميع التر اكيب الور اثية بالنسبة لطول الليفة فى الجنيتين و الموقعين. } \\
& \text { التحليل التجميعي جيزة } \\
& \text { أجرى التحليّل التجميعي بهدف تقدير تباين البيئات للجنية الأولى و الثانية بطريقتين المبانشرة وغير المبانرة. } \\
& \text { الطريقة المباشرة } \\
& \text { تعتمد على تحليل تجميعي للجنيتين الأولى و الثانية كل على حده وتم تقدير تباين البيئات. } \\
& \text { الطريقةغير المباشرة } \\
& \text { تعتمد على تحليل تجميعي للجنيتين الأولى و الثانية معا ثم تقسيم الجني الى الجنية الأولى و الثانية والاختلاف بينهما. }
\end{aligned}
$$

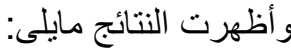

$$
\begin{aligned}
& \text { تفوق الطريقة غير المبانشرة على المبانشرة فى تقدير تباين البيئات للجنية الأولى والثانية حيث يتم ذلك فى تحليل تجميعي واحد }
\end{aligned}
$$

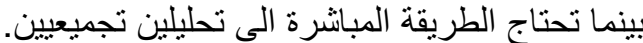

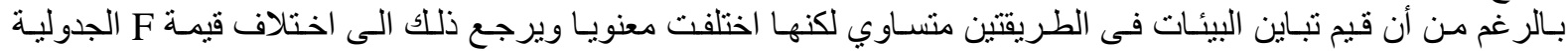

$$
\begin{aligned}
& \text { للطريقتين واختلاف قيم التباين البيئي للطريقينتين. }
\end{aligned}
$$

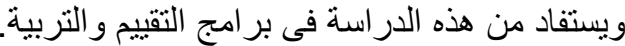

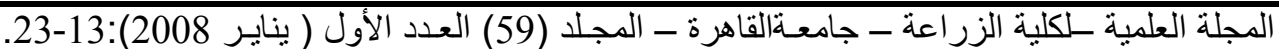

